\title{
Characterization of ion heat conduction in JET and ASDEX Upgrade plasmas with and without internal transport barriers
}

\author{
R C Wolf ${ }^{1}$, Y Baranov ${ }^{2}$, X Garbet ${ }^{3}$, N Hawkes ${ }^{2}$, A G Peeters ${ }^{4}$, C Challis ${ }^{2}$, \\ M de Baar ${ }^{5}$, C Giroud ${ }^{5}$, E Joffrin ${ }^{3}$, M Mantsinen 6 , D Mazon ${ }^{3}$, \\ H Meister $^{4}$, W Suttrop ${ }^{4}$, K-D Zastrow ${ }^{2}$ and the ASDEX Upgrade team \\ and contributors to the EFDA-JET Workprogramme \\ ${ }^{1}$ Institut für Plasmaphysik, Forschungszentrum Jülich, Association EURATOM/FZJ, Trilateral \\ Euregio Cluster, D-52425 Jülich, Germany \\ ${ }^{2}$ UKAEA/EURATOM Fusion Association, Culham Science Centre, Abingdon, OX14 3DB, UK \\ 3 Association EURATOM-CEA sur la fusion, CEA Cadarache, F-13108 St Paul lez Durance, \\ France \\ ${ }^{4}$ Max-Planck-Institut für Plasmaphysik, EURATOM-Assoziation, D-85748 Garching, Germany \\ ${ }^{5}$ FOM Instituut voor Plasmafyisica Rijnhuizen, Association EURATO-FOM, Trilateral Euregio \\ Cluster, PO Box 1207, 3430 BE Nieuwegein, The Netherlands \\ ${ }^{6}$ Helsinki University of Technology, Association-EURATOM Tekes, FIN-02015 HUT, Finland
}

Received 29 January 2003

Published 21 August 2003

Online at stacks.iop.org/PPCF/45/1757

\begin{abstract}
In ASDEX Upgrade and JET, the ion temperature profiles can be described by $R / L_{\mathrm{Ti}}$ which exhibits only little variations, both locally, when comparing different discharges, and radially over a wide range of the poloidal cross-section. Considering a change of the local ion heat flux of more than a factor of two, this behaviour indicates some degree of profile stiffness. In JET, covering a large ion temperature range from 1 to $25 \mathrm{keV}$, the normalized ion temperature gradient, $R / L_{\mathrm{Ti}}$, shows a dependence on the electron to ion temperature ratio or toroidal rotational shear. In particular, in hot ion plasmas, produced predominantly by neutral beam heating at low densities, in which large $T_{\mathrm{i}} / T_{\mathrm{e}}$ is coupled to strong toroidal rotation, the effect of the two quantities cannot be distinguished. Both in ASDEX Upgrade and JET, plasmas with internal transport barriers (ITBs), including the PEP mode in JET, are characterized by a significant increase of $R / L_{\mathrm{Ti}}$ above the value of $\mathrm{L}$ - and $\mathrm{H}$-mode plasmas. In agreement with previous ASDEX Upgrade results, no increase of the ion heat transport in reversed magnetic shear ITB plasmas is found in JET when raising the electron heating. Evidence is presented that magnetic shear directly influences $R / L_{\mathrm{Ti}}$, namely decreasing the ion heat transport when going from weakly positive to negative magnetic shear.
\end{abstract}




\section{Introduction}

In tokamak plasmas, the cross-field transport is generally above the predictions from neoclassical theory, which is based on the effect of binary collisions between the charged particles to explain the minimum transport level. This anomalous transport prevails in most parts of the plasma cross-section in both L- and H-mode. In contrast to the low confinement or L-mode, the high confinement or H-mode shows a confinement improvement localized at the plasma edge. The anomalous transport, is explained by various microinstabilities which drive plasma turbulence [Connor_94, Garbet_01] and thus enhance the cross-field transport. Internal transport barriers (ITBs) are regions in the core of the plasma, where, due to the partial or complete stabilization of these micro-instabilities, the anomalous transport is reduced or even suppressed [Wolf_03].

In regions of tokamak plasmas, which are dominated by anomalous transport, the normalized temperature gradient, $R / L_{\mathrm{T}}\left(R\right.$ : major radius, $L_{\mathrm{T}}=T /|\nabla T|$ : gradient length without sign, assuming that $\nabla T$ is always negative), changes little over a wide range of plasma parameters. This is observed for both electron [Hoang_01, Ryter_01] and ion heat transport [Peeters_00, Baker_01, Tardini_02]. This behaviour is often related to profile stiffness or profile resilience which is characterized by a reluctance of $R / L_{\mathrm{T}}$ to increase beyond a critical value despite a continuous increase of the applied heat flux. Various theoretical turbulence simulations predict the occurrence of such critical gradients, $R /\left.L_{\mathrm{T}}\right|_{\text {crit }}$, [Dimits_00] produced by a combination of ion temperature gradient (ITG) and trapped electron modes (TEMs) for the ion transport channel and TEMs and electron temperature gradient (ETG) modes for the electron transport channel.

Present research focuses on two questions. First, the question of profile stiffness per se is discussed. If it is accepted that in general the heat flux increases non-linearly with $R / L_{\mathrm{T}}$, the problem is reduced to the question whether a sharp transition at a certain value of $R /\left.L_{\mathrm{T}}\right|_{\text {crit }}$ from a weak to a strong dependence on the normalized gradient is observed. Second, a dependence of $R /\left.L_{\mathrm{T}}\right|_{\text {crit }}$ on plasma parameters may exist. Experimental $R / L_{\mathrm{T}}$ values show small changes, if the variation of the plasma parameters is large enough [Baker_01]. At least qualitatively this is in agreement with theory, which predicts various turbulence stabilization mechanisms, depending on plasma quantities, such as magnetic shear, $E \times B$ flow shear [Waltz_94, Waltz_95, Burrell_97, Waltz_98], ratio of electron to ion temperature, $T_{\mathrm{e}} / T_{\mathrm{i}}$, Shafranov shift and effective ion charge.

The existence of $R /\left.L_{\mathrm{T}}\right|_{\text {crit }}$ manifests itself in a strong increase of the heat flux once the critical gradient is exceeded. This behaviour is sketched in figure 1 , which illustrates different degrees of profile stiffness and the dependence of $R /\left.L_{\mathrm{T}}\right|_{\text {crit }}$ on plasma parameters. The degree of profile stiffness can be understood as the slope of the heat flux above the critical gradient. In this picture a completely stiff profile is described by a slope approaching infinity above $R /\left.L_{\mathrm{T}}\right|_{\text {crit }}$.

Assuming gyro-Bohm scaling of the heat conductivity, $\chi \sim T^{3 / 2} f\left(R / L_{\mathrm{T}}\right)$ [Connor_77], the conductive part of the heat flux is given by

$$
Q=-\chi n \nabla T \sim T^{5 / 2} n f\left(\frac{R}{L_{\mathrm{T}}}\right) .
$$

Hence, when investigating $f\left(R / L_{\mathrm{T}}\right)$, the normalization $Q / T^{5 / 2}$ will be used. The gyro-Bohm scaling of the heat conductivity is motivated by the assumption that the turbulence scale length is determined by the gyro-radius of the respective plasma species, in contrast to Bohm scaling, which also involves the macroscopic scale proportional to the dimension of the poloidal plasma cross-section. Especially for ions, the gyro-Bohm scaling may not apply below the threshold 
Degree of profile stiffness

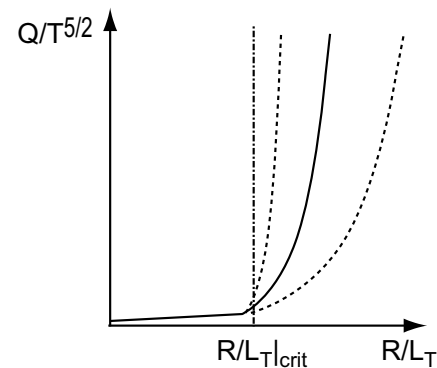

Variation with plasma parameters

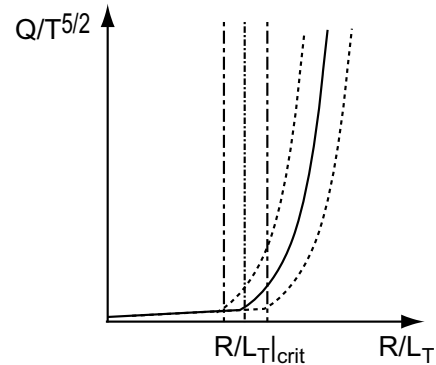

Figure 1. In the presence of stiff temperature profiles the heat flux increases sharply above the critical gradient $R /\left.L_{\mathrm{T}}\right|_{\text {crit }}$. The degree of profile stiffness is given by the slope of $Q / T^{5 / 2}$ above $R /\left.L_{\mathrm{T}}\right|_{\text {crit }}$ : the steeper the slope, the stronger the profile stiffness. At a given degree of profile stiffness $R /\left.L_{\mathrm{T}}\right|_{\text {crit }}$ may vary with plasma parameters.

$R /\left.L_{\mathrm{T}}\right|_{\text {crit }}$. If the ion transport dropped to neoclassical level, the scaling would be of the form $\chi \sim 1 / \sqrt{T}$ or $Q \sim \sqrt{T}$.

As mentioned before, the function $f\left(R / L_{\mathrm{T}}\right)$ in equation (1) is expected to depend on various plasma parameters (magnetic shear, $E \times B$ flow shear, $T_{\mathrm{e}} / T_{\mathrm{i}}$, etc). Therefore, ideally, one would measure the dependence of $Q$ on $R / L_{\mathrm{T}}$, keeping all other plasma quantities constant. Practically, this is rather difficult to achieve. The closest we could get in the study presented here is to evaluate $R / L_{\mathrm{T}}$ (or some representation of $R / L_{\mathrm{T}}$ ) at a given plasma radius for a large number of discharges, which cover significant changes of $Q$. To compensate for the influence of $E \times B$ flow shear and $T_{\mathrm{e}} / T_{\mathrm{i}}$, the dependence of $R / L_{\mathrm{T}}$ on these parameters will be considered. For ITB plasmas also the effect of magnetic shear is discussed.

A different type of representation is to take the radial profiles of $Q / T^{5 / 2}$ and $R / L_{\mathrm{T}}$ at a given time and plot them against each other. However, in this case, the interpretation with respect to profile stiffness is not straight forward, since plasma parameters change along the radial profiles and hence possibly also $R /\left.L_{\mathrm{T}}\right|_{\text {crit }}$. Assuming profile stiffness and little variations of $R /\left.L_{\mathrm{T}}\right|_{\text {crit }}$ with plasma parameters this representations indeed results in plots similar to figure 1, as has been obtained for electron [Ryter_01] and ion heat transport [Baker_01]. However, the inverse argument is not valid. The agreement with figure 1 does not necessarily imply profile stiffness. Also a disagreement could still be consistent with profile stiffness, provided the change of $R /\left.L_{\mathrm{T}}\right|_{\text {crit }}$ can be explained with the variation of plasma parameters along the radial profile.

As a consequence of temperature profile stiffness, the core temperature directly depends on the edge value (see equation (3)). In the simplified case of a radially constant density this results in a heat conductivity which is determined by the pressure at the plasma edge (e.g. pedestal pressure in the H-mode), given by the edge confinement, and the heat flux supplied to the plasma, $\chi(r) \propto-Q(r) / p_{\text {edge. }}$. Hence, provided the heat flux is large enough to reach the threshold above which the turbulence is excited, which limits the temperature profile to the critical gradient, a reduction of the heat conductivity does not necessarily indicate the appearance of an ITB. Of course, if the transport drops to neoclassical level in the plasma core, one would speak of an ITB. However, practically this is difficult to assess, since both the theoretical calculation of the neoclassical transport and the experimental determination of the heat conductivity are subject to considerable uncertainties [Peeters_00].

Therefore, assuming that the presence of turbulence which manifests itself in a critical gradient, $R /\left.L_{\mathrm{T}}\right|_{\text {crit }}$, which varies only little in L- and H-mode plasmas, an ITB can be 
characterized by the exceeding of this critical value [Peeters_00]. The advantage of this ITB definition, compared to the evaluation of absolute transport levels, is that it also holds in cases where, despite the formation of an ITB, the residual transport is still above neoclassical. This is in particular the case for the electron transport, which up to now seems to be always above neoclassical even in the presence of ITBs.

Also the $\rho^{*}$ criterion [Tresset_02], now frequently used at JET to characterize ITBs, contains the gradient length as an essential parameter:

$$
\rho_{\mathrm{T}}^{*}=\frac{\rho_{\mathrm{s}}}{L_{\mathrm{T}}}=\frac{\sqrt{m}}{Z e} \frac{\sqrt{T}}{B_{\phi}} \frac{\nabla T}{T}>\rho_{\mathrm{ITB}}^{*},
$$

where $m$ denotes the ion mass, $Z e$ the ion charge and $B_{\phi}$ the toroidal magnetic field. $\rho_{\mathrm{ITB}}^{*}$ is an empirical values (for JET $1.4 \times 10^{-2}$ ) which denotes the threshold above which an ITB is present. In contrast to this $\rho^{*}$ criterion, derived from the requirement that an ITB is present if the $E \times B$ shearing rate exceeds the maximum linear growth rate of the turbulence, the ITB definition based on $R /\left.L_{\mathrm{T}}\right|_{\text {crit }}$ does not include the dependence on the magnetic field or $\sqrt{T}$.

Although the considerations before apply to both electron and ion transport, this paper mainly focuses on ion transport. The electron temperature is considered only as far as the change of $T_{\mathrm{e}} / T_{\mathrm{i}}$ and its predicted effect on ITG mode stability is concerned. The following sections of the paper are organized as follows. First, some of the evidence for profile stiffness in ASDEX Upgrade and JET L- and H-mode plasmas will be discussed [Joffrin_02a]. ASDEX Upgrade and JET are both divertor tokamaks with a vertically elongated plasma cross-section. In its linear dimensions JET $(R=3.1 \mathrm{~m}$, minor radius $a \approx 0.8 \mathrm{~m})$ is about twice the size of ASDEX Upgrade $(R=1.65 \mathrm{~m}, a \approx 0.5 \mathrm{~m})$. The analysis presented here includes only stationary profiles. Heat modulation experiments [LopesC_95], which anyway are very difficult to perform for the ion heat transport, are beyond the scope of this paper. Second, the different types of ITBs observed in JET are characterized. This also includes a discussion of the effect of electron heating and magnetic shear.

Both in ASDEX Upgrade and JET the radial ion temperature profiles are measured by charge exchange recombination spectroscopy (CXRS) [Hellermann_90, Meister_01]. The profiles of the safety factor, $q$, are inferred from numerical equilibrium reconstructions (CLISTE in ASDEX Upgrade [McCarthy_99] and EFIT in JET [Lao_85]), constrained by motional-Stark-effect (MSE) measurements of the local magnetic field pitch angle [Wolf_97, Hawkes_99].

\section{L- and H-mode plasmas}

\subsection{ASDEX Upgrade}

An $R / L_{\mathrm{Ti}}$, which at a given radius does not depend on plasma conditions, is equivalent to ion temperature profiles which can be mapped onto each other with a constant multiplication factor. This is illustrated in figure 2(a) for two ASDEX Upgrade discharges, comparing an L- with an H-mode plasma. The same heating power of $5 \mathrm{MW}$ neutral beam injection (NBI) has been applied in both discharges. Plasma current and magnetic field are 1 MA and $2.5 \mathrm{~T}$ in both the L- and H-mode discharges. In the L-mode case, the transition from L- to H-mode has been prevented by limiting the plasma at the inner wall. Both discharges exhibit similarly low line averaged densities, ranging from $\bar{n}_{\mathrm{e}}=4.2 \times 10^{19} \mathrm{~m}^{-3}$ (in H-mode) to $4.7 \times 10^{19} \mathrm{~m}^{-3}$ (L-mode), which explains the comparatively high ion temperature values in the H-mode [Wolf_99]. Besides, the central ion temperature in the H-mode plasma is only slightly affected by magneto-hydro-dynamic (MHD) instabilities, as, in the absence of 
(a)

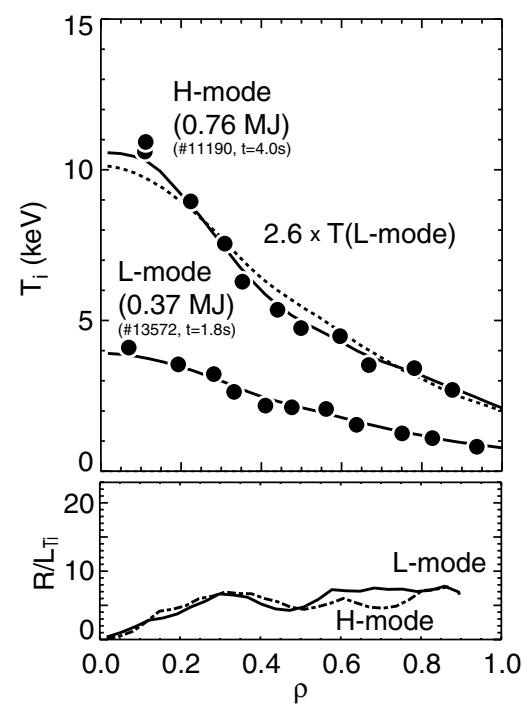

(b)

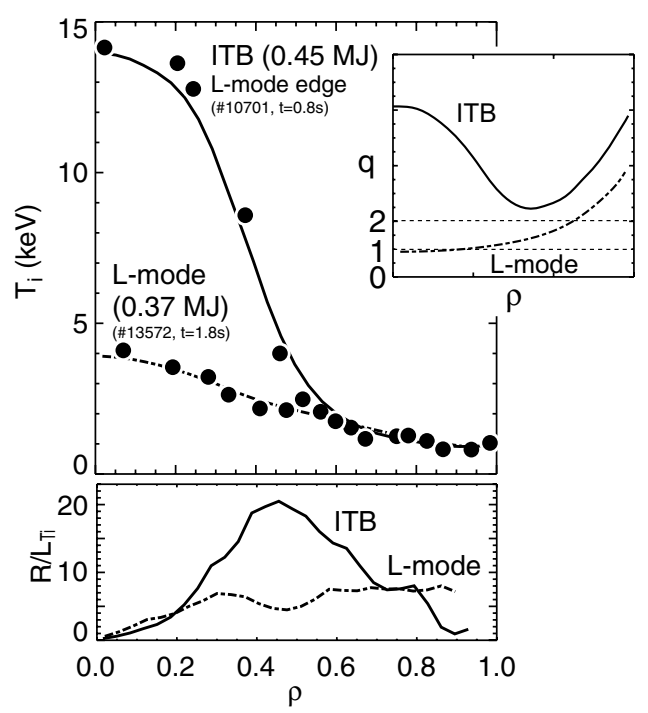

Figure 2. (a) The ion temperature profiles of two ASDEX Upgrade discharges with the same heating power of $5 \mathrm{MW}$ NBI are compared. At a constant $R / L_{\mathrm{Ti}}$ the difference in core temperature is caused by the different edge confinement of L- and H-mode. (b) An ITB, here with L-mode edge, is characterized by the exceeding of $R / L_{\mathrm{Ti}}$ of $\mathrm{L}$ - and $\mathrm{H}$-mode by a factor of approximately three. The ITB is generated by tailoring the $q$-profile, producing negative magnetic shear in the plasma centre. The lines in the temperature profile plots are spline fits to the measurements.

sawtooth oscillations, fishbone activity causes only little profile flattening. In the L-mode discharge sawtooth activity is present, but very weak. For the fishbones the central electron temperature changes from electron cyclotron emission, which in contrast to CXRS can resolve the effect of these fast MHD events, is less than 5\% [Guenter_99.pdf]. For the sawtooth oscillations the changes are about twice as large. Thus, also here, the influence of central MHD instabilities can be regarded as small. The H- and L-mode ion temperature profiles show essentially the same $R / L_{\mathrm{Ti}}$, also evident in the possibility to map the $\mathrm{L}$ - onto the $\mathrm{H}$-mode profile. The difference in plasma energy is a consequence of the different edge confinement and, to some extent, the larger density peaking in the H-mode [Peeters_02]. Figure 2(b) shows the corresponding case with ITB and L-mode edge. As illustrated by the insert, the ITB is generated by tailoring the $q$-profile producing negative magnetic shear in the plasma centre (for a discussion of ITBs see later).

For the three sets of profiles shown in figure $2, Q / T_{\mathrm{i}}^{5 / 2}$ is plotted as a function of $R / L_{\mathrm{Ti}}$ in figure 3 . The fact that $R / L_{\mathrm{Ti}}$ is constant not only locally when comparing $\mathrm{L}$ - and $\mathrm{H}$-mode, but also does not change over most of the plasma cross-section, is also seen here. Above a certain value of $Q / T_{\mathrm{i}}^{5 / 2}$, which is different for $\mathrm{L}$ - and $\mathrm{H}$-mode, $R / L_{\mathrm{Ti}}$ remains constant at about 6 (figures $3(a)$ and $(b)$ ). The ITB plasma in contrast shows a strong excursion of $R / L_{\mathrm{Ti}}$ approaching 20 at low $Q / T_{\mathrm{i}}^{5 / 2}$ (figure $3(c)$ ).

In regions where $R / L_{\mathrm{Ti}}$ is below approximately 6 (corresponding to radii $\rho \leqslant 0.4$ ) the slopes of the L- and H-mode curves become similar if, instead of gyro-Bohm scaling, the temperature dependence of the neoclassical heat transport is used (see second row of plots in figure 3). This is in agreement with the results from the power balance analysis of the ion heat conductivity, which shows that for both L- and H-mode the ion heat transport in the central 

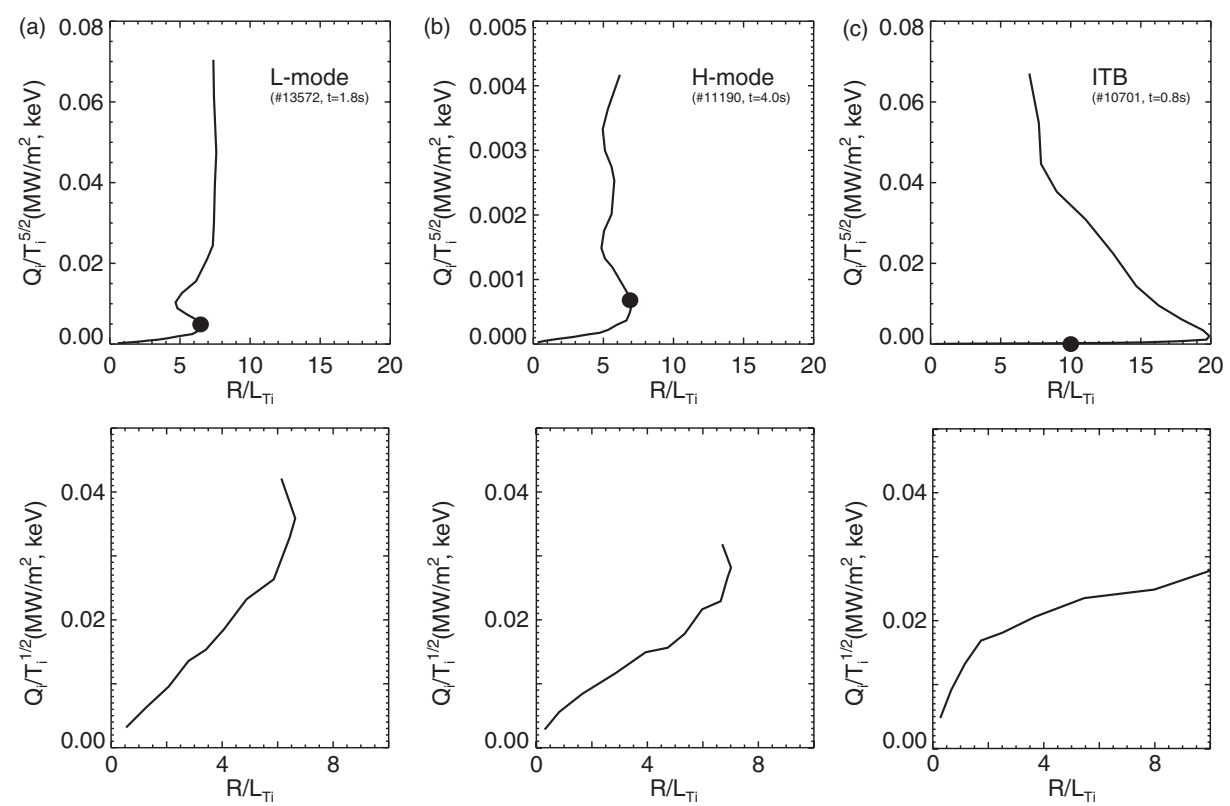

Figure 3. $Q / T_{\mathrm{i}}^{5 / 2}$ as a function $R / L_{\mathrm{Ti}}$ for the three ASDEX Upgrade cases shown in figure 2. The plots have been obtained from the radial profiles of the respective quantities, covering a radial range of $0 \leqslant \rho \leqslant 0.8$ (top row). $Q$ has been evaluated by the transport code ASTRA [Pereverzev_02]. In (a) $\mathrm{L}$ - and $(b) \mathrm{H}$-mode $R / L_{\mathrm{Ti}}$ does not depend on $Q / T_{\mathrm{i}}^{5 / 2}$ above a certain threshold. (c) In the ITB, in contrast, this value of $R / L_{\mathrm{Ti}} \approx 6$ is clearly exceeded. For $R / L_{\mathrm{Ti}}$ below 6 , i.e. $\rho \leqslant 0.4$, the plots in the bottom row show a different scaling, $Q / T_{\mathrm{i}}^{1 / 2}$, corresponding to the temperature dependence of the neoclassical transport. Note, here the same vertical scale has been used for the three cases. The dots in the upper row of plots indicates the range covered by the bottom row.

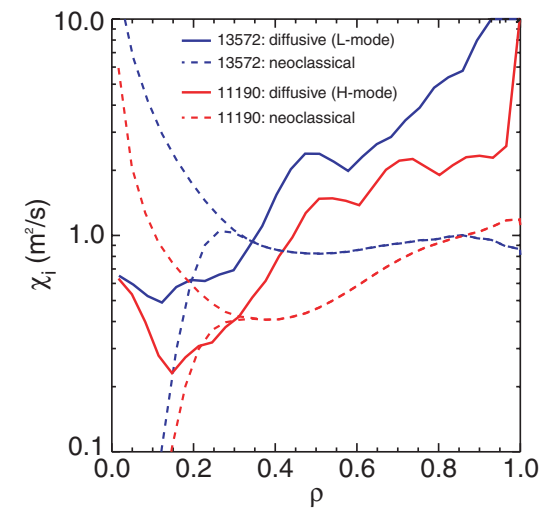

Figure 4. Ion heat conductivities, inferred from the transport code ASTRA [Pereverzev_02], of the L- and $\mathrm{H}$-mode plasmas shown in figure 2(a). For the comparison with the neoclassical ion heat conductivities two cases, with [Lin_97] and without [Chang_86] orbit width corrections in the plasma centre, are shown.

region of the plasma is of the order of the neoclassical transport (figure 4). Of course one should bear in mind that especially in the plasma centre without a strong, well defined central heat source the error margins of the power balance analysis can be very large. In case of the ITB also the neoclassical scaling, $Q / T_{\mathrm{i}}^{1 / 2}$, deviates from the $\mathrm{L}$ - and $\mathrm{H}$-mode cases which, 


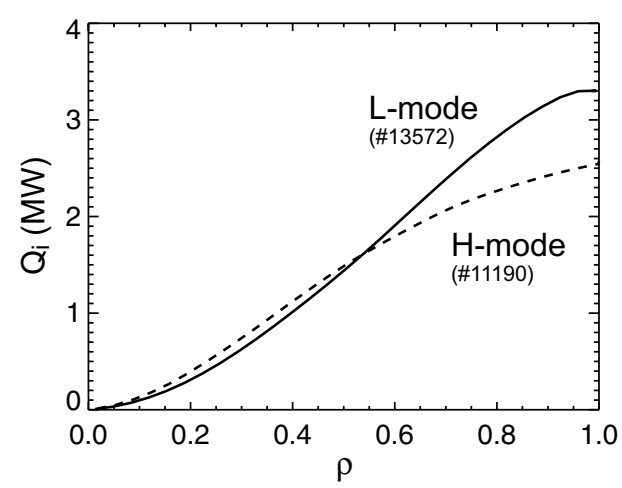

Figure 5. Ion heat flux profiles (diffusive), inferred from the ASTRA code [Pereverzev_02], for the L- and H-mode plasmas shown in figure $2(a)$.

however, is not surprising, as for the ITB plasma also the safety factor differs considerably. As shown in figure 2(b) the $q$-profile is monotonic in the L-mode (and also H-mode), while in the ITB discharge the magnetic shear is negative in the plasma centre with a $q$-profile minimum of $q_{\min } \approx 2$.

As a result of the same heating power and similar plasma parameters (plasma current: 1.0 MA, toroidal magnetic field: $2.5 \mathrm{~T}$, density: $\bar{n}_{\mathrm{e}}=4.2 \times 10^{19} \mathrm{~m}^{-3}$ in H-mode and $4.7 \times 10^{19} \mathrm{~m}^{-3}$ in L-mode) also the ion heat flux profiles of the L- and $\mathrm{H}$-mode plasmas differ only little over much of the plasma cross-section (figure 5). Only towards the plasma edge the L-mode starts to lie above the H-mode, reaching a total flux which is about $30 \%$ higher. The density profiles are affected by the different confinement which may be the cause for the remaining differences of $Q$. From the heat fluxes shown, assuming the same heat conductivity in $\mathrm{L}$ - and $\mathrm{H}$-mode and allowing for the larger pedestal temperature of the $\mathrm{H}$-mode, the expected core temperature of the H-mode would be higher by only $50 \%$ and not by a factor 2.6 , as observed in the experiment. This, together with an unchanged $R / L_{\mathrm{Ti}}$, is an indication for profile stiffness.

A convenient representation of the ion temperature gradient length or $R / L_{\mathrm{Ti}}$ is to express the central ion temperature, $T_{\mathrm{i}}\left(\rho_{1}\right)$ as a function of the temperature at a radial position closer to the plasma edge, $T_{\mathrm{i}}\left(\rho_{2}\right)$ [Peeters_00]

$$
T_{\mathrm{i}}\left(\rho_{1}\right)=T_{\mathrm{i}}\left(\rho_{2}\right) \cdot \exp \left(\varepsilon \int_{\rho_{1}}^{\rho_{2}} \frac{R}{L_{\mathrm{Ti}}} \mathrm{d} \rho\right) .
$$

$\varepsilon=a / R$ is the inverse aspect ratio and $\rho=r / a$ the normalized minor radius. The gradient in $\nabla T_{\mathrm{i}}$ is taken at the outer midplane. In the subsequent analysis, the normalized radii $\rho_{1}=0.2$ and $\rho_{2}=0.6$ have been chosen, as this region basically covers the range where $R / L_{\mathrm{Ti}}$ does not change. Thus, a constant gradient length results in a linear dependence of $T_{\mathrm{i}}\left(\rho_{2}\right)$ on $T_{\mathrm{i}}\left(\rho_{1}\right)$

$$
T_{\mathrm{i}}\left(\rho_{1}\right)=T_{\mathrm{i}}\left(\rho_{2}\right) \cdot \exp \left(\varepsilon \Delta \rho \frac{R}{L_{\mathrm{Ti}}}\right),
$$

where $\Delta \rho=\rho_{2}-\rho_{1}$.

In ASDEX Upgrade L- and $\mathrm{H}$-mode discharges follow a straight line in this representation of the ion temperature profiles, covering a range of central ion temperatures from about 2 to $10 \mathrm{keV}$. The corresponding L- and $\mathrm{H}$-mode $R / L_{\mathrm{Ti}}$ lies at 6 [Tardini_02] in agreement with the $R / L_{\mathrm{Ti}}$ seen in figure 3 . 


\subsection{JET}

At first, the $R / L_{\mathrm{Ti}}$ of $20 \mathrm{JET}$ discharges is inferred according to equation (4) by applying a linear fit to the ion temperature points of each discharge separately. Since the ion temperature is measured by CXRS, this procedure only includes times when NBI is applied to the plasma. The discharges have been selected to cover an ion temperature range as large as possible. This covers the early L-mode phases of the discharges and also the high temperature phases of edge localized mode (ELM) free H-modes (also called hot ion H-modes) [Rimini_99], in which central $T_{\mathrm{i}}$ values of up to $25 \mathrm{keV}$ have been reached.

An example is shown in figure 6. JET pulse 52096 is taken from a series of discharges where by changing the electron density and the ratio of NBI to ion cyclotron resonance heating (ICRH), keeping the total heating power constant, the electron heat flux is varied, in order to investigate the electron temperature profile stiffness [Suttrop_01]. It is evident that the variation of $R / L_{\mathrm{Ti}}$, both in $\mathrm{L}$ - and $\mathrm{H}$-mode, is rather small in this case. The $R / L_{\mathrm{Ti}}$ values, derived in the same manner for all the $20 \mathrm{JET}$ discharges, is summarized in figure 7 . Obviously the variation

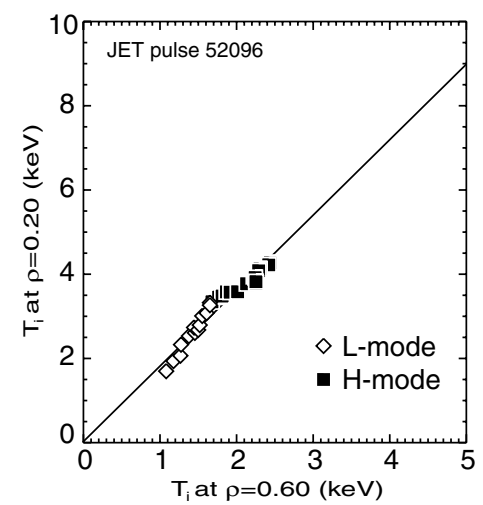

Figure 6. $T_{\mathrm{i}}(0.2)$ versus $T_{\mathrm{i}}(0.6)$ for JET pulse 52096. The fit to the data points yields $R / L_{\mathrm{Ti}}=5.7 \pm 0.5$. The variation of $R / L_{\mathrm{Ti}}$ during both $\mathrm{L}$ - and $\mathrm{H}$-mode phases (with type-III ELMs) is rather small.

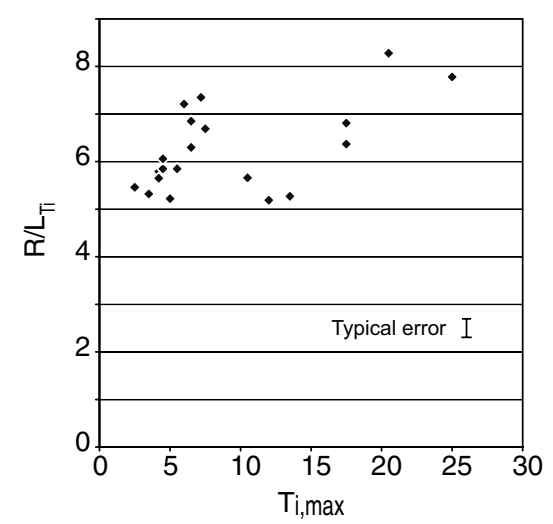

Figure 7. $R / L_{\mathrm{Ti}}$ as a function of the maximum ion temperature, $T_{\mathrm{i}, \max }$, at $\rho=0.2$ for the $20 \mathrm{JET}$ discharges, selected for the analysis. In most cases, the variation of $R / L_{\mathrm{Ti}}$ between discharges is larger than the one within a discharge. The typical error from the fit of the individual discharges is also shown. 
between the different discharges is larger, although, as can be seen in the ASDEX Upgrade example in figure $2(b)$ and will be shown later also for JET, it is still small compared to the changes observed in ITB plasmas.

In order to account for the possible influence of $T_{\mathrm{e}} / T_{\mathrm{i}}$ or the $E \times B$ shearing rate, $\omega_{E \times B}$, on the ion transport, the representation of $T_{\mathrm{i}}\left(\rho_{1}\right)=f\left(T_{\mathrm{i}}\left(\rho_{2}\right)\right)$ is modified as follows:

$$
T_{\mathrm{i}}\left(\rho_{1}\right)=T_{\mathrm{i}}\left(\rho_{2}\right) \cdot \exp \left(\varepsilon \Delta \rho \frac{R}{L_{\mathrm{Ti}}}+c_{1}\left(\frac{T_{\mathrm{i}}}{T_{\mathrm{e}}}-1\right)+c_{2} \frac{\omega_{E \times B}}{\gamma_{\mathrm{ITG}}}\right),
$$

where $c_{1}$ and $c_{2}$ are parameters to be inferred from the experimental data. Here, the maximum linear growth rate is approximated by

$$
\gamma_{\mathrm{ITG}}=\frac{v_{\mathrm{i}, \mathrm{th}}}{\sqrt{R L_{\mathrm{Ti}}}}
$$

where $v_{\mathrm{i}, \mathrm{th}}$ is the ion thermal velocity. In addition, $\omega_{E \times B}$ is assumed to be dominated by the toroidal rotational shear, $\partial v_{\phi} / \partial r$,

$$
\omega_{E \times B}=\frac{r}{q R} \frac{\partial v_{\phi}}{\partial r}
$$

where $q \approx 1$ is the safety factor and $r$ the minor radius [Waltz_94]. According to this reference, such sheared flows have a stabilizing influence in tokamak plasmas, where the Kelvin-Helmholtz instability appears to be compensated by the combined effects of magnetic shear and $E \times B$ flow shear. In a tokamak like JET, where the toroidal rotation is driven by unidirectional NBI, studies of the composition of $\omega_{E \times B}$ confirm the validity of the approximation given in equation (7) in many cases [Maget_02]. Work by [Ernst_98] and more recently by [Petty_02] indicate that indeed the plasma core can be affected by $E \times B$ shear without necessarily showing an ITB (see also discussion about ELM free H-modes later). For the calculation of $T_{\mathrm{e}} / T_{\mathrm{i}}$ and $\omega_{E \times B}$ the values of the respective quantities are taken at $\rho=0.4$. Hereby, $T_{\mathrm{e}}$ is measured by LIDAR Thomson scattering and $v_{\phi}$ by CXRS.

In addition, to avoid any complication due to the influence of density gradients on the ion transport, points with large values of $R / L_{\mathrm{ne}}$ ( $L_{\mathrm{ne}}$ : electron density gradient length) are excluded in the following considerations. In the absence of a high quality density profile diagnostic with high enough spatial resolution, the measured density line integrals normalized to the length of their lines of sight through the plasma are used to derive $R / L_{\text {ne }}$

$$
\frac{R}{L_{\text {ne }}}=\frac{\int_{1} n_{\mathrm{e}} \mathrm{d} l / l_{1}-\int_{2} n_{\mathrm{e}} \mathrm{d} l / l_{2}}{1 / 2\left(\int_{1} n_{\mathrm{e}} \mathrm{d} l / l_{1}+\int_{2} n_{\mathrm{e}} \mathrm{d} l / l_{2}\right)} \frac{R}{\Delta R} .
$$

$\Delta R$ denotes the radial distance of the poloidal tangent radii of the respective lines of sight (the normalized tangent radii $r / a$ are approximately 0.1 and 0.6 ). Using this as a measure for the density gradient length, only data points with $R / L_{\mathrm{ne}} \leqslant 0.75$ have been included in the analysis of L- and H-modes. For the ITBs (see later) this restriction has not been made. An example of a density profile with $R / L_{\mathrm{ne}} \approx 0.75$ is given in figure 8 . Although this definition of $R / L_{\mathrm{ne}}$ reflects relative trends quite well, the absolute value is not necessarily in agreement with conventionally calculated density gradient lengths.

The pairs of ion temperatures of all 20 discharges are plotted in figure 9 with and without the correction for $T_{\mathrm{e}} / T_{\mathrm{i}}$ and $\omega_{E \times B}$, i.e. the open symbols simply represent the measured temperature profiles and the closed symbols are derived from the measured values, applying equation (5) to the core temperature values at $\rho_{1}$.

Besides the exclusion of data points where $R / L_{\mathrm{ne}}$ is large, the representation of the ion temperature profiles without this correction is the same as for the individual discharges, an example of which is shown in figure 6. In this case, the fit to the data yields $R / L_{\mathrm{Ti}}=6.8$, 


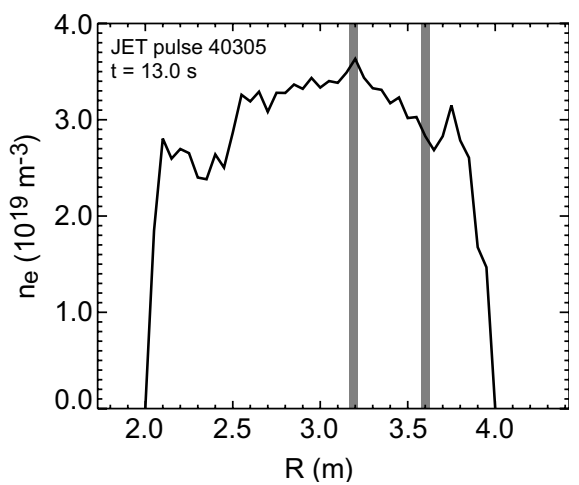

Figure 8. Example of a density profile (from LIDAR Thomson scattering) which, according to the definition given in equation $(8)$, has an $R / L_{\mathrm{ne}} \approx 0.75$. The tangent radii of the line integrals are also indicated.

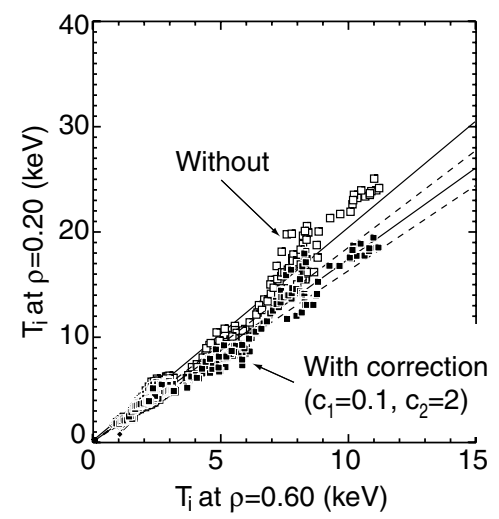

Figure 9. $T_{\mathrm{i}}\left(\rho_{1}\right)$ as a function of $T_{\mathrm{i}}\left(\rho_{2}\right)$ for the 20 discharges also underlying figure 7. Accounting for effects of $T_{\mathrm{e}} / T_{\mathrm{i}}$ and $\partial v_{\phi} / \partial r$ on $R / L_{\mathrm{Ti}}$ achieves a better fit at high $T_{\mathrm{i}}$. The dashed lines correspond to ten times the standard deviation of the fit to the temperature points.

but does not describe the data at high $T_{\mathrm{i}}$ very well. The ion temperature points tend to deviate from a straight line at higher $T_{\mathrm{i}}$. With the correction according to equation (5) the reduction of the core ion temperature improves the linear fit at high $T_{\mathrm{i}}$. The resulting $R / L_{\mathrm{Ti}}$ becomes

$$
5.3+\frac{0.1\left(T_{\mathrm{i}} / T_{\mathrm{e}}-1\right)}{(\varepsilon \cdot \Delta \rho)}+\frac{2 \omega_{E \times B} / \gamma_{\mathrm{ITG}}}{(\varepsilon \cdot \Delta \rho)} .
$$

It is worth noting that the effect of $T_{\mathrm{e}} / T_{\mathrm{i}}$ and $\partial v_{\phi} / \partial r$ cannot be easily distinguished, as in plasmas dominated by unidirectional NBI heating, raising $T_{\mathrm{i}}$ above $T_{\mathrm{e}}$ also means increasing the toroidal rotation. This is also reflected in the approximately linear scaling of $v_{\phi}$ with $T_{\mathrm{i}}$ [Maget_02]. Thus, one could also choose $c_{1}=0.2, c_{2}=0$ (only taking $T_{\mathrm{e}} / T_{\mathrm{i}}$ in to account) or $c_{1}=0, c_{2}=4$ (only taking $\partial v_{\phi} / \partial r$ into account) and achieve a similarly good description of the points at high ion temperature. To make a distinction between the two contributions to $R / L_{\mathrm{Ti}}$, an experiment is required which predominantly heats the ions without increasing the toroidal rotation.

Coming back to the question of profile stiffness, it is already evident from figure 9 that similar to ASDEX Upgrade [Peeters_00] the JET L- and H-mode ion temperature profiles can 

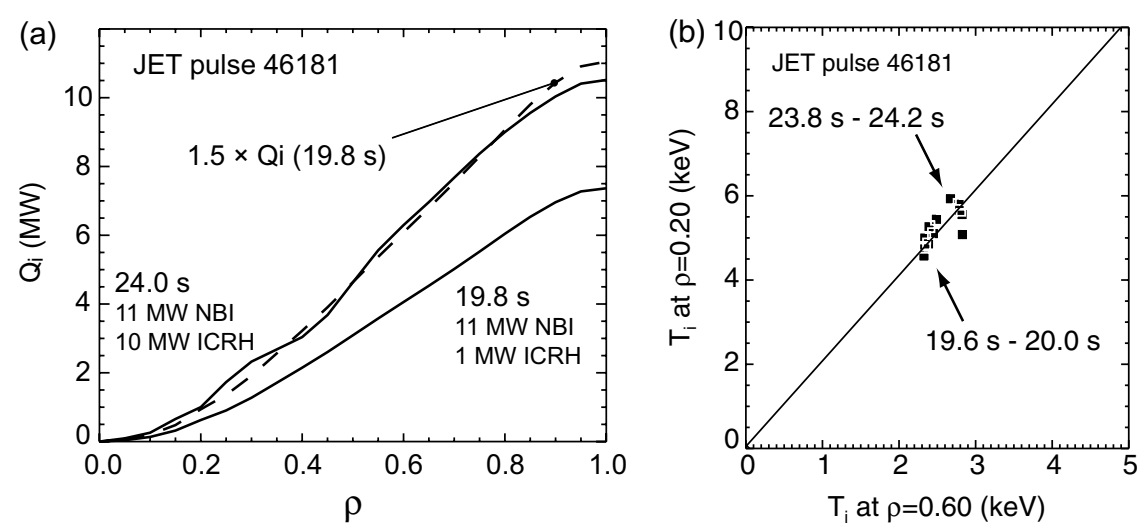

Figure 10. (a) The ion heat fluxes for a JET type-I ELMy H-mode (pulse 46181), inferred from the PION code [Eriksson_93] is shown for two different combinations of ICRH and NBI powers at 19.8 and $24.0 \mathrm{~s}$. The additional ICRH results in an increase of the ion heat flux by $50 \%$. $(\mathrm{b})$ Despite this increase, the ion temperature level increases only marginally (by $10 \%$ ), while $R / L_{\mathrm{Ti}}$ stays constant.

be described by a common $R / L_{\mathrm{Ti}}$, in JET including dependencies on $T_{\mathrm{e}} / T_{\mathrm{i}}$ or $\omega_{E \times B}$. For the ASDEX Upgrade discharges in figure 2(a) it has been shown that, despite similar heat fluxes, the absolute ion temperatures at constant $R / L_{\mathrm{Ti}}$ can be considerably different, caused by a different edge confinement. The JET data set contains a complementary example where, despite differing heat fluxes the ion temperature profiles are almost the same (figure 10). First, the discharge is heated with a combination of $1 \mathrm{MW}$ ICRH and $11 \mathrm{MW}$ NBI. A type-I ELMy H-mode develops. Subsequently, the ICRH power is raised to $10 \mathrm{MW}$ at constant density, increasing the ion heat flux by $50 \%$. Assuming a constant heat conductivity one would expect also a corresponding increase of the ion temperature, which however is not seen. The actual $T_{\mathrm{i}}$ increase (core and edge) at constant $R / L_{\mathrm{Ti}}$ is only $10 \%$. Apparently, the profile is clamped at a critical value of $R / L_{\mathrm{Ti}}$ and the ion temperature does not follow the increase of the heat flux, since the edge confinement changes only marginally, which is a further indication for profile stiffness.

For a subset of the discharges presented in figure 9 the ion heat flux profiles have been calculated by the PION code [Erikson_93]. To a large extent, these discharges originate from an experiment in which the electron heat flux was varied to study electron transport [Suttrop_01]. Additional discharges are the one shown in figures 10 and 19. Altogether, the temperature range of the $\mathrm{H}$-mode plasmas (shown in figure 9) from about 2 to $20 \mathrm{keV}$ (at $\rho=0.4$ ) is covered. In figure 11, these ion heat flux values, taken at $\rho=0.4$ and normalized to $T_{\mathrm{i}}^{5 / 2}$, are plotted against $R / L_{\mathrm{Ti}}$ and $R / L_{\mathrm{Ti}} \times T_{\mathrm{e}} / T_{\mathrm{i}}$, respectively. As already known from the discussion before, $R / L_{\mathrm{Ti}}$ changes only little. The underlying variation of the ion heat flux is more than a factor of two which (according to figure 1) suggests some degree of profile stiffness. However, the weakness of this picture is that plasma parameters expected to influence $R / L_{\mathrm{Ti}}$, such as magnetic shear, $T_{\mathrm{e}} / T_{\mathrm{i}}$ and $\omega_{E \times B}$ are not kept constant (for the effect of magnetic shear see also later). A more qualitative attempt to rectify the effect of $T_{\mathrm{e}} / T_{\mathrm{i}}$ is made in figure 11 by multiplying $R / L_{\mathrm{Ti}}$ with $T_{\mathrm{e}} / T_{\mathrm{i}}$. Despite the remaining scatter of the data points, which to some extent may be caused by the uncertainties of the spatial derivatives of the ion temperature profiles, a trend similar to the one shown in figure 1 can be seen.

The subsequent discussion is based on the assumption that $\mathrm{L}$ - and $\mathrm{H}$-mode plasmas are to some degree critical gradient limited and that this critical gradient, $R /\left.L_{\mathrm{Ti}}\right|_{\text {crit }}$, can be used 


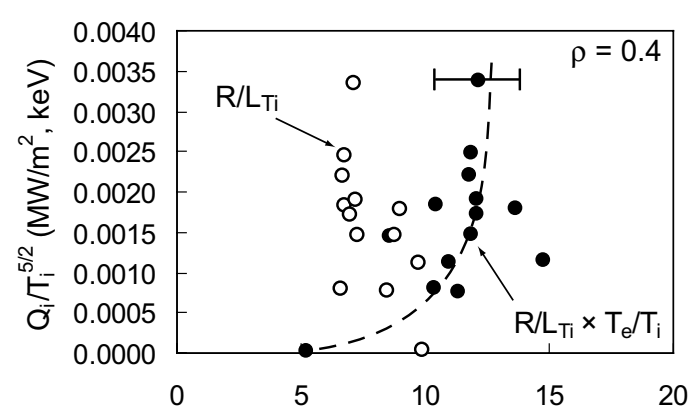

Figure 11. From a subset of the discharges shown in figure 9 the ion heat flux (at $\rho=0.4$ ) is calculated by the PION code [Eriksson_93] and, normalized to $T_{\mathrm{i}}^{5 / 2}$, plotted versus $R / L_{\mathrm{Ti}}(\mathrm{O})$. To account for the effect of changes of the electron to ion temperature ratio $Q_{\mathrm{i}} / T_{\mathrm{i}}^{5 / 2}$ is also plotted versus $R / L_{\mathrm{Ti}} \times T_{\mathrm{e}} / T_{\mathrm{i}}(\bigcirc)$. The dashed line (not a fit) indicates a trend similar to the one depicted in figure 1 .

to characterize such plasmas. Although not entirely comprehensive, the evidence presented earlier certainly points in that direction.

\section{Characterization of ITBs}

In ASDEX Upgrade, internal barriers of the ion heat transport are easily identified by comparing the ratio $T_{\mathrm{i}}\left(\rho_{1}\right) / T_{\mathrm{i}}\left(\rho_{2}\right)$ between ITB and L- or H-mode plasmas [Peeters_00]. All reversed magnetic shear ITBs and high- $\beta_{\mathrm{p}}$ plasmas [Hobirk_01] lie above the $R / L_{\mathrm{Ti}}$ for L- and $\mathrm{H}$-modes in ASDEX Upgrade. In fact, this criterion led to the conclusion that, in contrast to original claims that stationary H-modes with ITBs have been produced in ASDEX Upgrade [Wolf_99], these plasmas do not exhibit internal barriers of the ion heat transport [Peeters_02]. The ASDEX Upgrade H-mode discharge described in the previous section (ASDEX Upgrade pulse 11190) is such an example. Despite the low ion heat conductivity in the plasma core and the large ion temperature gradients, the $R / L_{\mathrm{Ti}}$ is still not different from L-mode. This discussion, however, does not include particle transport and whether the density peaking in these discharges is an indication of some type of internal barriers or not is a different question.

To investigate the same dependence in JET, the JET ITB scenarios are classified into three types: (a) the optimized shear (OS) regime, corresponding to target $q$-profiles with low but positive magnetic shear [Sips_98], (b) the reversed shear (RS) regime with a region of negative magnetic shear in the plasma centre [Challis_01] and (c) the pellet enhanced performance (PEP) mode, where the central shear reversal is produced by the combination of pellet injection and strong central heating [Hugon_92].

The corresponding plots of $T_{\mathrm{i}}\left(\rho_{1}\right)=f\left(T_{\mathrm{i}}\left(\rho_{2}\right)\right)$ are shown in figure 12 for 23 OS discharges, 17 RS discharges and 3 PEP modes. Since the radial range is limited to $\rho=0.6$, ITBs which are broader are excluded. The temperature data include the ITB formation phase just after the NBI has been switched on, the time period during a fully established ITB and in some cases also the time period when the ITB decays. The latter can be caused by a transition of the plasma edge to type-I ELMs or in the case of the PEP mode by the decay of the density peaking. With the PEP modes also a very recent example is included (JET pulse 55882), where the ITB is facilitated by a sequence of first lower hybrid current drive to produce the magnetic shear reversal, then a string of pellets injected into the core of the plasma and finally NBI and 

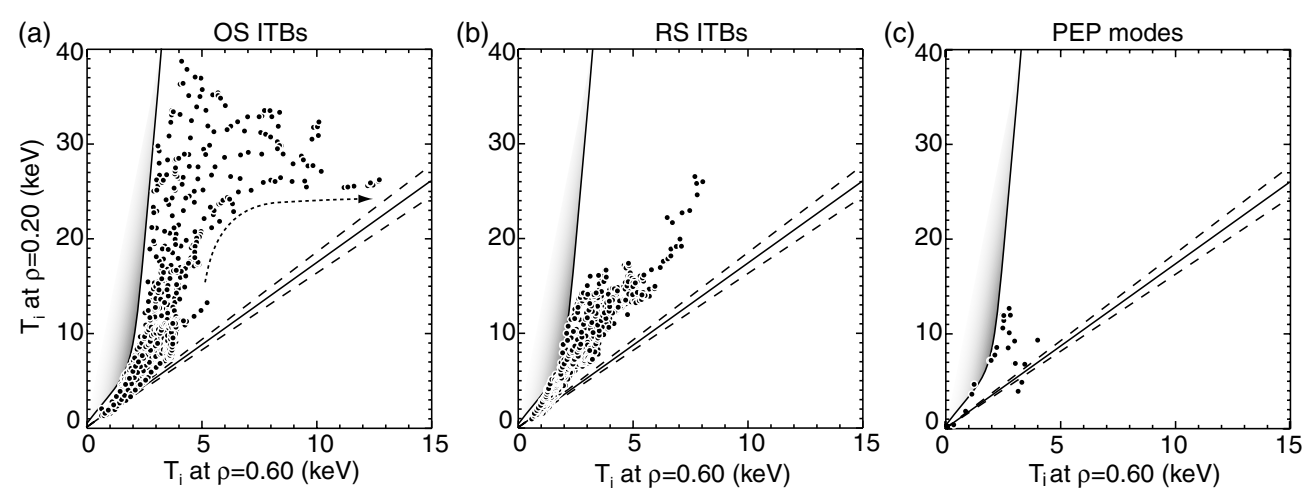

Figure 12. $T_{\mathrm{i}}\left(\rho_{1}\right)=f\left(T_{\mathrm{i}}\left(\rho_{2}\right)\right)$ for three types of JET ITB discharges, OS plasmas (23 discharges), RS plasmas (17 discharges) and PEP modes ( 3 discharges). The straight line corresponds to the fit of the $\mathrm{L}$ - and $\mathrm{H}$-mode reference cases shown in figure $9\left(R / L_{\mathrm{Ti}}=5.3\right)$. All ion temperature points of the different ITB plasmas lie at or above this reference. Besides the lower limit given by $R / L_{\mathrm{Ti}}=5.3$, an upper limit is indicated by the shaded area. The dashed line in $(a)$ shows the effect of a rise of the pedestal $T_{\mathrm{i}}$.

ICRH to form a high density ITB. As a reference $R / L_{\mathrm{Ti}}=5.3$ from the analysis of $\mathrm{L}$ - and $\mathrm{H}$-mode plasmas (illustrated in figure 9) is taken.

Before the formation of the ITB, at low heating power and low $T_{\mathrm{i}}$, or after its collapse, $R / L_{\mathrm{Ti}}$ agrees with this reference value. When an ITB is formed, this value is exceeded considerably, reaching $R / L_{\mathrm{Ti}}$ up to 18 . It is also evident that in this picture, where an ITB is characterized by an increase of $R / L_{\mathrm{Ti}}$ above a critical value, the PEP mode qualifies as an ITB also for ions. Owing to the high plasma density, $T_{\mathrm{i}}$ is generally much lower in the PEP modes. In addition, the high collisional coupling of ions and electrons facilitates $T_{\mathrm{e}} \approx T_{\mathrm{i}}$ without the need of comparable amounts of auxiliary ion and electron heating as in low density ITBs [Forest_96, Wolf_00].

Besides the lower limit given by $R / L_{\mathrm{Ti}}=5.3$, there is also an upper limit which, however, does not follow a $R / L_{\mathrm{Ti}}=$ const. curve. At present, one can only speculate, whether this limit corresponds to neoclassical ion transport, an anomalous transport process, different from those considered so far, or an ideal MHD limit. Many of the ITBs with the largest pressure gradients are known to be very close to ideal MHD instabilities, which often are the cause for the disruptive termination of the discharges [Huysmans_99]. However, the radial extent of the associated MHD modes is rarely local enough to merely limit the pressure gradient. Instead, the MHD modes tend to become global, leading to the destruction of the ITB confinement. Also the fact that the upper limit is far from a $\nabla T_{\mathrm{i}}=$ const. curve, which is of the form $T_{\mathrm{i}}\left(\rho_{1}\right)=T_{\mathrm{i}}\left(\rho_{2}\right)+\partial T_{\mathrm{i}} / \partial \rho \Delta \rho$, makes a pressure driven MHD limit, given by a maximum $\nabla p$, unlikely.

Another interesting observation is that, in the ITB plasmas investigated, the increase of the pedestal temperature, due to the transition of the plasma edge from $\mathrm{L}$ - to $\mathrm{H}$-mode or type-III to type-I ELM confinement, is rarely accompanied by a rise of the central ion temperature. In most cases either the core $T_{\mathrm{i}}$ stays constant or drops slightly. This is most visible in the OS cases, where the increase of $T_{\mathrm{i}}\left(\rho_{2}=0.6\right)$ can result in a reduction of $R / L_{\mathrm{Ti}}$ to almost the level of L- and H-modes (dashed line in figure 12(a)). Such a behaviour is in contrast to the general believe that the gain in plasma energy due to the formation of an ITB is simply adding to the energy, $W_{\mathrm{H}} \approx p_{\text {ped }} V$, given by the $\mathrm{H}$-mode pedestal pressure, $p_{\text {ped }}$, and the plasma volume, $V$. However, depending on the ITB radius the difference between 
the two possibilities in terms of plasma energy is not necessarily very large. For an ITB at half radius the difference between an ITB adding to the pedestal pressure or not would be $\frac{1}{4} p_{\text {ped }} V$ only.

\subsection{Influence of electron heating}

In plasmas with ITBs different observations have been made regarding the effect of electron heating on confinement. Central counter-current drive, provided by either electron cyclotron resonance heating $(\mathrm{ECH})$ and current drive in ASDEX Upgrade [Wolf_00] or by fast wave heating (FWH) and current drive in DIII-D [Forest_96], to support the negative magnetic shear in NBI heated ITB discharges, does not have any detrimental influence on the plasma confinement. In the case of DIII-D, this is partially attributed to the turbulence stabilizing effect of an increase of the $E \times B$ shearing rate due to a reduction of the poloidal magnetic field in the plasma centre, caused by the additional bootstrap current of the electron pressure. Although such an explanation is also conceivable for the ASDEX Upgrade case, the stabilizing effect of the additional Shafranov shift seems to be sufficient to compensate the destabilization of the ITG modes due to the increase of $T_{\mathrm{e}} / T_{\mathrm{i}}$. In contrast, in DIII-D ITB discharges with low or slightly negative central shear the addition of pure $\mathrm{ECH}$ or FWH leads to confinement degradation in both the ion and electron channel [Greenfield_99, Staebler_98].

In JET, a corresponding experiment has been devised to investigate the influence of electron heating in reversed magnetic shear ITB discharges, using ICRH at a low hydrogen minority concentration to maximize the power fraction going into the electrons. Starting from a negative central shear configuration, produced with off-axis LHCD in co-current direction during the current ramp-up phase, plasmas with NBI heating only are compared with plasmas with combined NBI and ICRH.

A comparison of two discharges is presented in figure 13. In one discharge $12 \mathrm{MW} \mathrm{NBI}$ only is applied and in the other a combination of $10 \mathrm{MW} \mathrm{NBI}$ and $6 \mathrm{MW}$ central ICRH. At first, a weak ITB forms, which is followed by a transition into a strong ITB phase at $6 \mathrm{~s}$, clearly visible on the neutron signal and the larger $R / L_{\mathrm{Ti}}$ (figure 15). In both discharges, the ITB phase is terminated by the appearance of strong ELM activity, which at the high power level can be avoided only transiently.

The main effect of the replacement of $2 \mathrm{MW}$ NBI by $6 \mathrm{MW}$ ICRH is an energy increase, which corresponds to a rise of the $H$-factor by $15 \%$. The neutron rate increases only marginally, which is reflected in the very small rise of $T_{\mathrm{i}}$ at constant density, while the $T_{\mathrm{e}}$-profile mainly broadens. This behaviour is confirmed by the calculation of the heat flux from TRANSP [Budny_95] using the PION code [Eriksson_93] for the ICRH power deposition (shown in figure 14). While the ion heat flux hardly changes, as the reduction of the NBI contribution is compensated by the ICRH, the electron heat flux increases by more than $40 \%$.

In both cases at $5.5 \mathrm{~s}$ the profiles of the ion and electron heat conductivity are very similar, which is in qualitative agreement with the comparable $H$-factors. After the transition to the strong ITB phase, the increase of $H_{\text {ITER89-P }}$ is reflected in a shift of the low electron and ion heat conductivity values in the plasma core to larger radii, concluding that the additional electron heat flux causes a broadening of the high confinement region. Currently, we can only speculate, whether the absence of a confinement degradation with electron heating is a consequence of the relatively strong shear reversal produced with LHCD in JET or whether other turbulence stabilizing effects such as the increase of the Shafranov shift contribute.

A power scan from $10 \mathrm{MW}$ NBI only to $18 \mathrm{MW}$ of combined NBI and ICRH shows, that both the time of the transition to the strong ITB and the gradient lengths after this transition are independent of the power level and the partition between electron and ion heat flux (figure 15). 
(a)
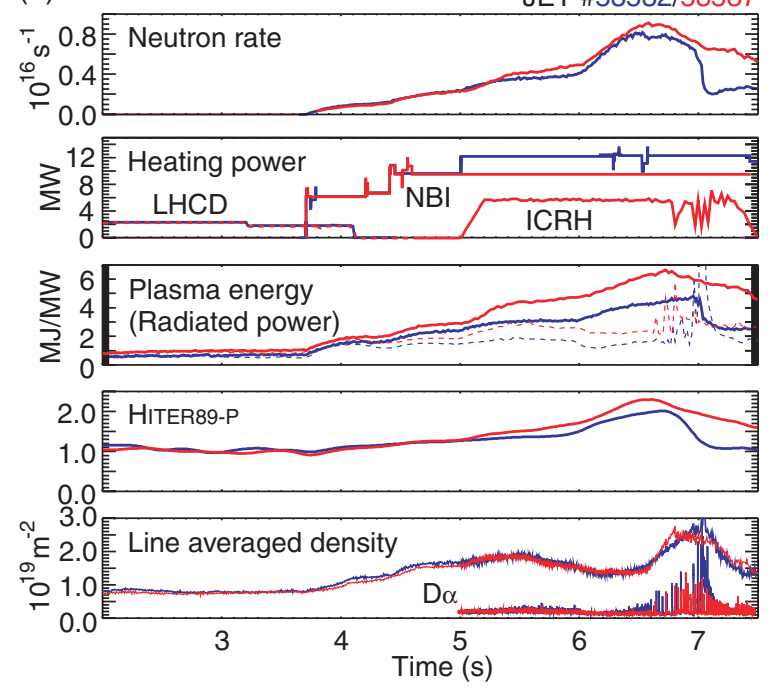

(b) $\quad 6.5 \mathrm{~s}$
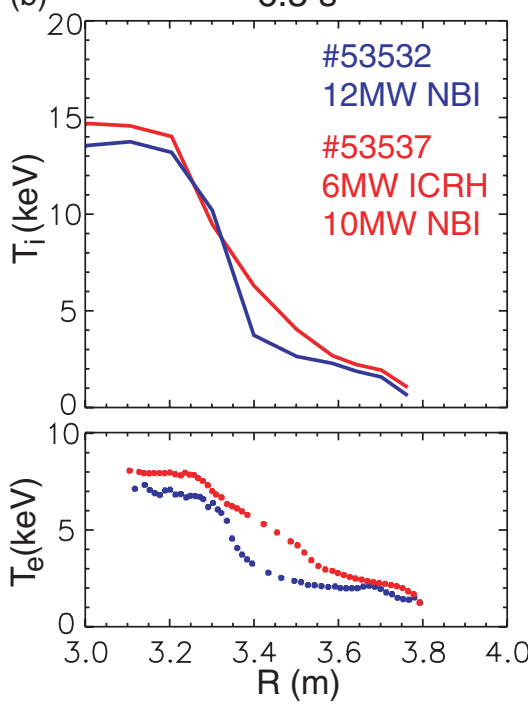

Figure 13. ITB plasmas with reversed magnetic shear (2.2 MA and 2.6 T), generated by LHCD during the current ramp, which ends at $4.7 \mathrm{~s}$. (a) Shows the time evolution of neutron rate, heating power, plasma energy, radiated power, $H$-factor $\left(H_{\text {ITER89-P }}\right)$, line averaged density, and $\mathrm{D}_{\alpha}$-signal. (b) Shows the temperature profiles at $6.5 \mathrm{~s}$, after the transition into a strong internal barrier.
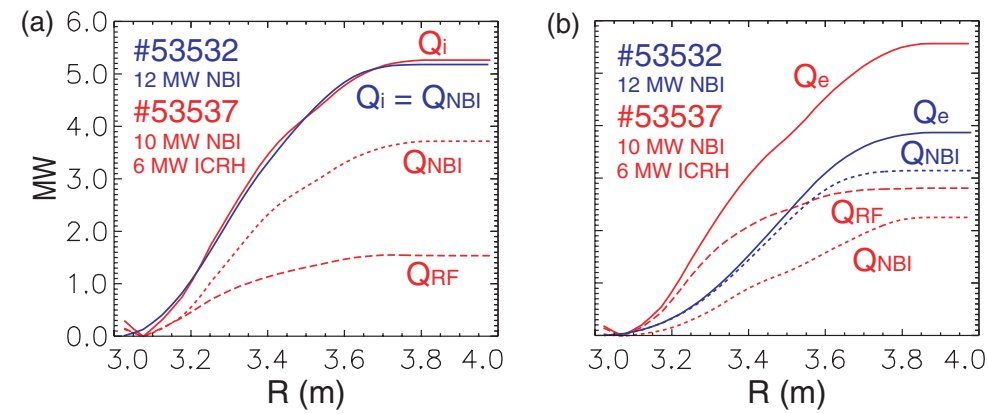

Figure 14. Composition of $(a)$ ion and $(b)$ electron heat flux from TRANSP [Budny_95] at $6 \mathrm{~s}$ for the two discharges shown in figure 13 just prior to the transition to the strong ITB phase (in contrast to the previous definition, here the heat flux $Q$ is not normalized to the flux surface area). The ICRH power deposition has been inferred from the PION code [Eriksson_93]. The ion heat flux hardly changes, as the reduction of the NBI contribution is compensated by ICRH. In contrast, the electron heat flux increases by more than $40 \%$.

Only the temperature at which the transition takes place increases with power. This endorses the conclusion that at least the level of electron heating produced with $6 \mathrm{MW} \mathrm{ICRH}$ does not lead to a degradation of the ion confinement. However, caution is advised when generalizing this statement to higher ICRH power, since in JET $6 \mathrm{MW}$ ICRH is just the power level required to reach the critical electron gradient length in H-mode plasmas [Suttrop_01]. Supposedly $6 \mathrm{MW}$ ICRH is not enough to excite TEMs, a higher power would cause a profound change of ITG mode stability. 


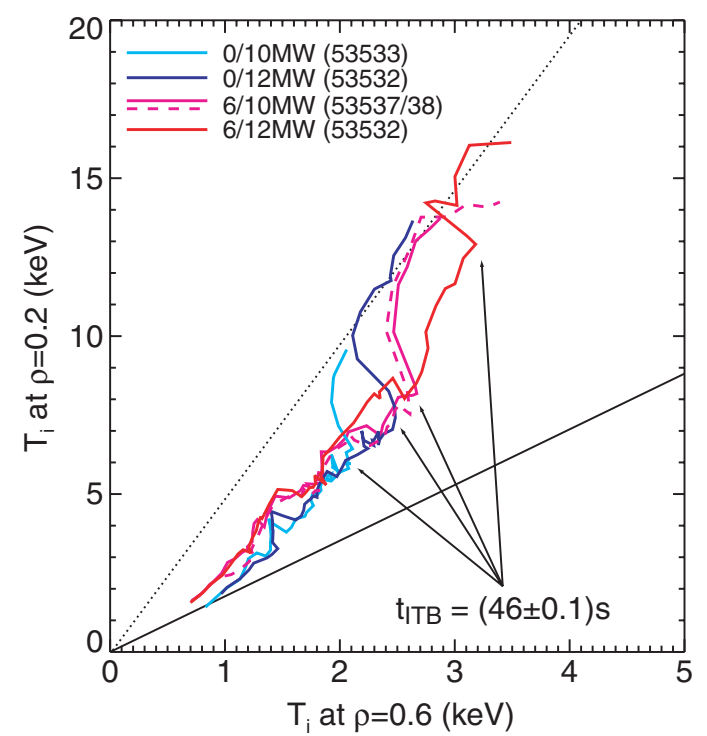

Figure 15. Time trajectories of $T\left(\rho_{1}\right)=f\left(T\left(\rho_{2}\right)\right)$ for different power levels and heating mixtures. Indicated are the ratios of ICRH to NBI heating power. The time evolution of the various discharges (2.2 MA, 2.6 T) resembles those shown in figure 13. First, a weak ITB is formed. The subsequent strong ITB phases exhibit a larger $R / L_{\mathrm{Ti}}$, indicated by the dotted line. The solid line represents the reference $R / L_{\mathrm{Ti}}$ of $\mathrm{L}$ - and $\mathrm{H}$-mode plasmas. The strong ITBs exhibit the same $R / L_{\mathrm{Ti}}$ independent of the power level and heating mixture. Also the time of the transition to the strong ITB, $t_{\mathrm{ITB}}$, is more or less independent of the power level.

\subsection{Effect of magnetic shear}

The main difference between the OS and RS ITBs in JET is the shape of the $q$-profile. Tailoring the $q$-profile with the early application of LHCD to heat the electrons, thus reducing the current diffusion, and to drive off-axis current to support the formation of negative magnetic shear in the plasma core [Hawkes_01], the power threshold of the ITB formation could be reduced considerably [Becoulet_01]. This suggests in line with theoretical considerations that the magnetic shear plays an essential role in the reduction or suppression of the plasma turbulence.

Figure 16 compares an OS with a RS discharge. The operational parameters of the discharges are similar (OS/pulse 51860, 2.8 MA, 2.6 T, $10 \mathrm{MW}$ NBI, 5 MW ICRH; RS/pulse 53537, 2.2 MA, 2.6 T, 10 MW NBI, 6 MW ICRH) except for the $q$-profile, which in the first case is monotonic with $q_{0} \approx 1$ and in the second shows negative central shear with $q_{\min } \approx 2$. Both discharges develop an H-mode edge with type-III ELMs and for the time periods shown also pulse 51860 does not exhibit sawtooth oscillation despite the low central $q$. Further details of pulse 51860 are described elsewhere [Joffrin_02a]. The main difference with respect to the evolution of $R / L_{\mathrm{Ti}}$ is that the OS plasma approximately follows the $R / L_{\mathrm{Ti}}$ of $\mathrm{L}$ - and $\mathrm{H}$-mode ion temperature profiles before forming an ITB, while the RS plasma immediately departs from this value. Later in the course of the discharge a second transition to an even higher $R / L_{\mathrm{Ti}}$ is observed, which earlier has been referred to as strong ITB.

The different ion transport properties of the two discharges can also be seen on the dependence of the normalized heat flux on $R / L_{\mathrm{Ti}}$. The curve in figure $17(a)$ for pulse 51860 before the ITB formation resembles those of the ASDEX Upgrade L- and H-modes, which show a constant $R / L_{\mathrm{Ti}}$ over large parts of the profiles (figures $3(a)$ and $(b)$ ). The difference 


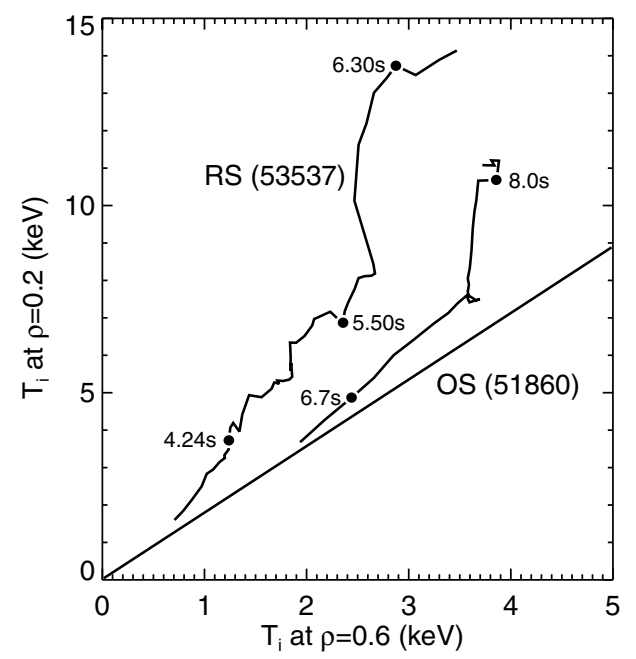

Figure 16. Time trajectories of a JET OS and a RS discharge. For the discussion several time points are marked. Except for the $q$-profile the plasma parameters of both discharges are similar. While the RS plasma immediately departs from the L- and H-mode reference, the OS follows this $R /\left.L_{\text {Ti }}\right|_{\text {crit }}$ line before forming an ITB.
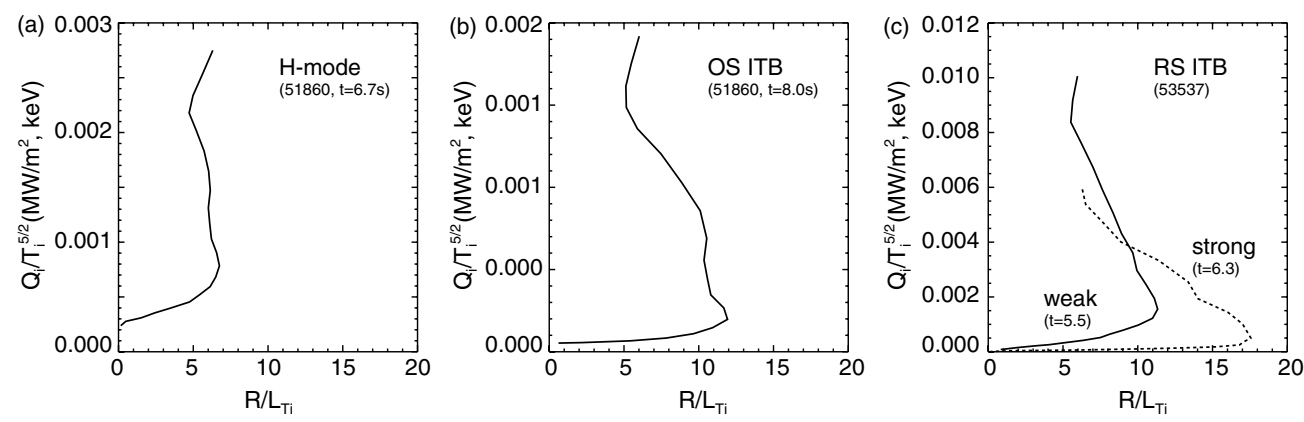

Figure 17. $Q / T_{\mathrm{i}}^{5 / 2}$ as a function $R / L_{\mathrm{Ti}}$ for the two JET OS and RS discharges shown in figure 16 . The Plots have been obtained from the radial profiles of the respective quantities, covering a radial range of $0 \leqslant \rho \leqslant 0.7$. $Q$ has been evaluated using TRANSP [Budny_95]. (a) Similar to ASDEX Upgrade, without an ITB a constant $R / L_{\mathrm{Ti}}$ over large parts of the profile is observed. Both in (b) the OS and (c) the RS case ITBs are characterized by large deviations from this $R / L_{\mathrm{Ti}}$ at low $Q / T_{\mathrm{i}}^{5 / 2}$.

between the OS ITB (figure 17(b)) and the weak and strong RS ITBs (figure 17(c)) is more of quantitative nature. All three profiles clearly deviate from the constant $R / L_{\mathrm{Ti}}$ values, reaching $R / L_{\mathrm{Ti}}$ up to 18 during the strong ITB of the RS case.

In order to clarify the role played by the magnetic shear in the ITB formation, the dependence of the critical $R / L_{\mathrm{Ti}}$ has been calculated by the gyro-kinetic turbulence code GS2 [Kotschenr_95] for linear stability. Since here the intention is to recover only the principle dependencies, the calculations have been performed assuming large aspect ratio, a circular plasma cross-section and $T_{\mathrm{e}} \approx T_{\mathrm{i}}$. Non-adiabatic electrons have been included (the values of perpendicular wave number times ion gyro-radius and safety factor are $k_{\perp} \rho_{\mathrm{i}}=0.3$ and $q=1) . \quad E \times B$ shear flow effects have not been considered. The results are shown in figure 18. In qualitative agreement with other such transport simulations [e.g. Wakatani_98] 


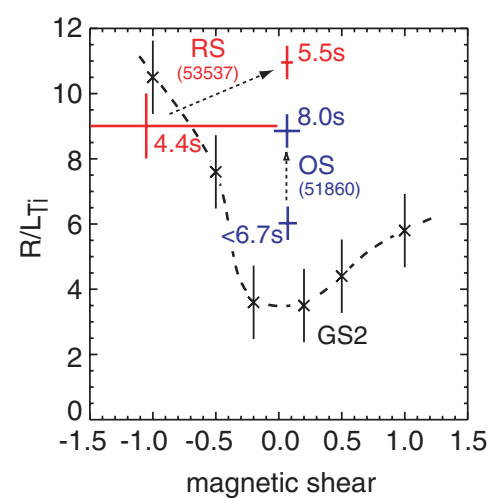

Figure 18. Theoretical calculations of the dependence of the critical $R / L_{\mathrm{Ti}}$ on the magnetic shear by the GS2 code [Kotchenreuther_95] for linear stability. For both positive and negative magnetic shear a increase of $R / L_{\mathrm{Ti}}$ is predicted. Also shown are the $R / L_{\mathrm{Ti}}$ and magnetic shear values of two JET discharges. The magnetic shear from EFIT and MSE is shown for the RS discharge (pulse no 53537) at 4.4 and $5.5 \mathrm{~s}$ and the OS discharge (pulse no 51860) before $(<6.7 \mathrm{~s})$ and after $(8.0 \mathrm{~s})$ the formation of an ITB.

the achievable $R / L_{\mathrm{Ti}}$ increases for both positive and negative magnetic shear with a minimum close to zero magnetic shear. However, the ballooning representation in GS2 does not allow the details at $s=0$ to be resolved.

In the RS discharge at $4.4 \mathrm{~s}$ the magnetic shear at the position of the ITB, given by the radius of the maximum of $R / L_{\mathrm{Ti}} \approx 10$, is approximately $-1 \pm 1$. The limited accuracy of MSE and CXRS data does not allow the value to be specified more precisely. In the OS discharge before the ITB formation $\left(R / L_{\mathrm{Ti}} \approx 6\right)$, the magnetic shear at $\rho=0.4$, which corresponds to the average radial position of the time trajectory in figure 16 , is between 0 and 0.1 . Since in this discharge MSE data are only available before the full NBI power is applied, the evolution of the magnetic shear has to be inferred from Faraday rotation polarimetry or current diffusion calculations. Both indicate that the $q$-profile is not changing very much. The much larger error of the magnetic shear of the RS case results from the strong local variation of $s$ with radius near the position of the ITB.

Comparing the experimental values of $R / L_{\mathrm{Ti}}$ and magnetic shear with the theoretical predictions (shown in figure 18) it is feasible that the magnetic shear is the cause for the bifurcation of the RS discharge from the L- and H-mode reference immediately after ion heating is applied. However, this would mean that at least initially also the RS discharge is limited by a critical gradient, which is only increased due to the effect of the negative magnetic shear. Later in the RS discharge $(5.5 \mathrm{~s})$ the ITB $\left(R / L_{\mathrm{Ti}} \approx 11\right)$ moves to larger radii into the region of zero or even slightly positive magnetic shear, requiring other mechanisms such as $E \times B$ shear to sustain the ITB. The OS plasma before the formation of the ITB exhibits a $R / L_{\mathrm{Ti}}$ which is higher than the theoretical calculation, which is not surprising considering the simplifications made. Obviously, as the magnetic shear does not change significantly when the ITB is formed in the OS plasma, this also must involve other turbulence stabilization mechanisms.

\subsection{Is the JET ELM free, hot ion H-mode an ITB?}

As discussed earlier, JET ELM free, hot ion H-mode discharges [Rimini_99] have been included in the determination of $R / L_{\mathrm{Ti}}$ values of $\mathrm{L}$ - and $\mathrm{H}$-mode plasmas. Accounting for the increase 


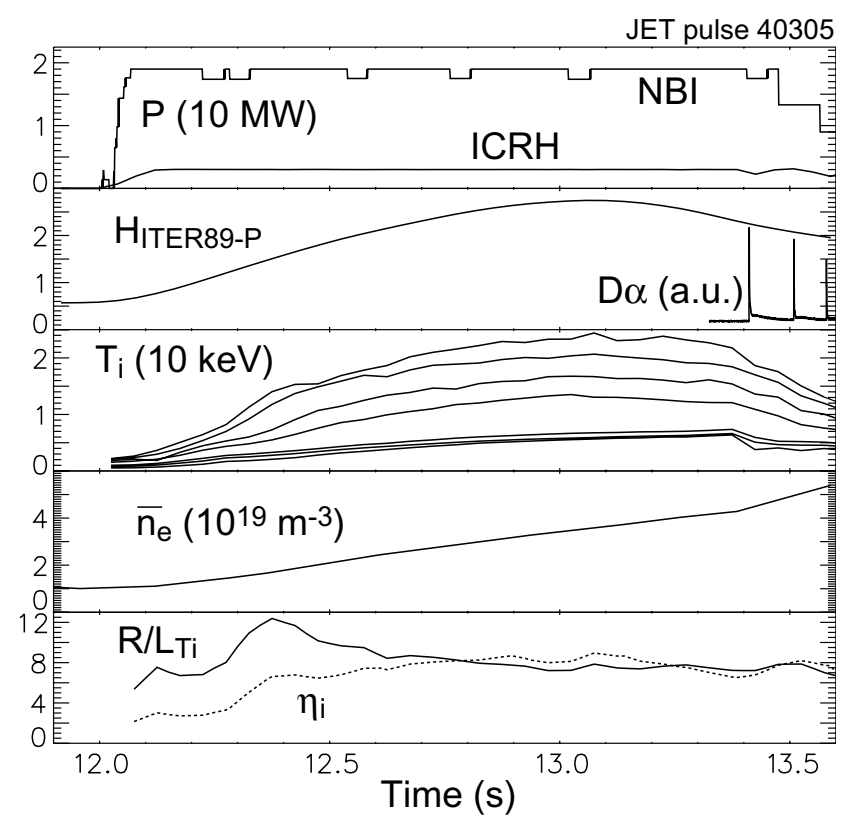

Figure 19. Time evolution of an ELM free H-mode in JET, showing NBI and ICRH powers, $H$-factor $\left(H_{\text {ITER89-P }}\right), \mathrm{D}_{\alpha}$ trace, ion temperature traces along the radial profile from centre to edge, line averaged density $\left(\bar{n}_{\mathrm{e}}\right)$ and normalized temperature gradients, $R / L_{\mathrm{Ti}}$ and $\eta_{\mathrm{i}}=L_{\mathrm{ne}} / L_{\mathrm{Ti}}$ (both evaluated at $\rho \approx 0.4$ ). After the application of NBI and ICRH at low density the ion temperature rises rapidly before the high confinement is finally terminated by the appearance of ELMs.

of $R / L_{\mathrm{Ti}}$ due to decreases of $T_{\mathrm{e}} / T_{\mathrm{i}}$ or increases of $\partial v_{\phi} / \partial r$, both assumed to be turbulence stabilizing and excluding temperature profiles which coincide with higher values of $R / L_{\mathrm{ne}}$, these discharges are comparable with the other $\mathrm{H}$ - and L-mode plasmas. Consequently, the high confinement is consistent with a critical $R / L_{\mathrm{Ti}}$ value and a large pedestal pressure, obtained in the absence of edge pressure limiting MHD instabilities. However, during the temporal evolution of ELM free H-modes, in some examples $R / L_{\mathrm{Ti}}$ transiently increases which cannot be attributed to the influence of $T_{\mathrm{e}} / T_{\mathrm{i}}$ or $\partial v_{\phi} / \partial r$.

Such a case (JET pulse 40305) is illustrated in figure 19. After the application of NBI and ICRH at low density the ion temperature rises rapidly before the high confinement is finally terminated by the appearance of strong ELMs. The time trace of $R / L_{\mathrm{Ti}}$ shows a strong excursion which coincides with an increased $R / L_{\mathrm{ne}}$, while the criticality parameter $\eta_{\mathrm{i}}=L_{\mathrm{ne}} / L_{\mathrm{Ti}}$ stays constant. This suggests that $R / L_{\mathrm{Ti}}$ is influenced by changes of the density gradient. For this reason, to avoid the influence of density gradients, the analysis of the JET L- and H-mode plasmas discussed earlier has been restricted to low values of $R / L_{\mathrm{ne}}$.

The different causes for variations of $R / L_{\mathrm{Ti}}$ are also reflected in the dependence $Q / T_{\mathrm{i}}^{5 / 2}$ on $R / L_{\mathrm{Ti}}$, shown in figure 20 , which compares times with high $\left(R / L_{\mathrm{ne}} \approx 2.0\right)$ and lower density peaking $\left(R / L_{\mathrm{ne}} \approx 1.0\right)$. At the early time $(12.35 \mathrm{~s})$, when $R / L_{\mathrm{Ti}}$ reaches its maximum, an excursion from a constant $R / L_{\mathrm{Ti}}$ similar to those with ITBs can be seen (figure 20(a)). Also multiplying $R / L_{\mathrm{Ti}}$ with $T_{\mathrm{e}} / T_{\mathrm{i}}$ to account for the influence of ion temperatures, which are higher than electron temperatures, does not remove this structure. This is in contrast to the later time point (13.2 s), when the plasma energy has reached its maximum and the curve resembles more 

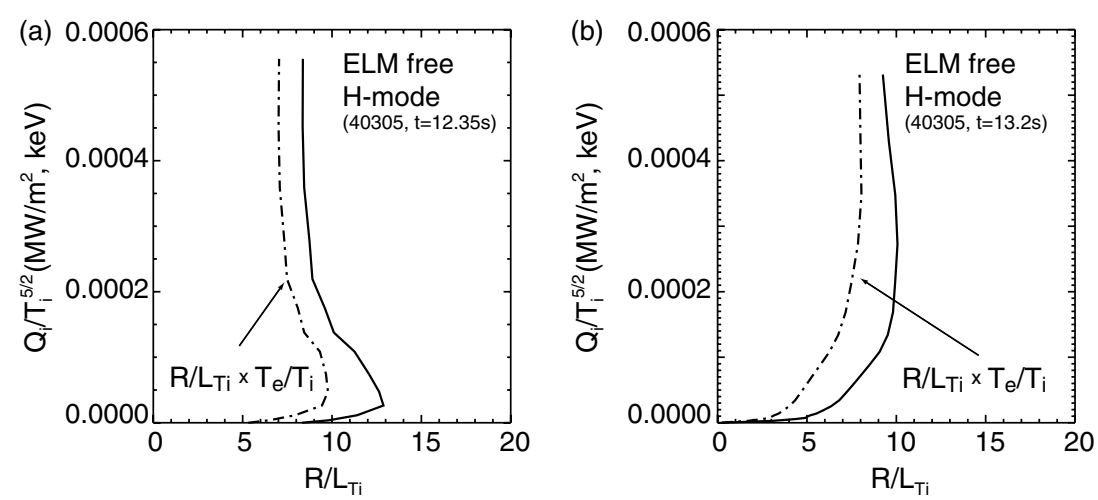

Figure 20. $Q / T_{\mathrm{i}}^{5 / 2}$ as a function $R / L_{\mathrm{Ti}}$ for the ELM free H-mode discharges shown in figure 19 (JET pulse 40305). The plots have been obtained from the radial profiles of the respective quantities, covering a radial range of $0 \leqslant \rho \leqslant 0.7$. (a) At $12.35 \mathrm{~s}$ the excursion of $R / L_{\mathrm{Ti}}$ cannot be explained by a lower $T_{\mathrm{e}} / T_{\mathrm{i}}$, but coincides with strong density peaking, $R / L_{\mathrm{ne}} \approx 2.0$. (b) Later at $13.5 \mathrm{~s}$, when the density peaking is lower, $R / L_{\mathrm{ne}} \approx 1.0$, the curve resembles that of low temperature $\mathrm{H}$-modes, especially when accounting for $T_{\mathrm{e}} / T_{\mathrm{i}}$ effects.

that of a typical H-mode (figure $20(b)$ ). Here, the multiplication with $T_{\mathrm{e}} / T_{\mathrm{i}}$ not only reduces $R / L_{\mathrm{Ti}}$ to a value observed in low temperature H-modes, but also decreases the small local deviation towards higher $R / L_{\mathrm{Ti}}$.

From this one can conclude that at low $R / L_{\text {ne }}$ ion temperature profiles in the JET ELM free $\mathrm{H}$-mode can be characterized by a $R / L_{\mathrm{Ti}}$ which depends on the electron to ion temperature ratio (or the toroidal rotational shear) in the same way as for all other $\mathrm{H}$-mode plasmas. At high density peaking, the situation is not so clear. The larger $R / L_{\mathrm{Ti}}$, which lies well in the range of ITB plasmas, indicates the presence of an ITB. On the other hand, the approximately constant $\eta_{\mathrm{i}}$ suggests that the ion temperature profiles still can be described by a critical gradient, if $R / L_{\mathrm{Ti}}$ is replaced by $\eta_{\mathrm{i}}$.

\section{Summary and conclusions}

The ion heat transport in both ASDEX Upgrade [Peeters_00, Tardini_02] and JET L- and $\mathrm{H}$-mode discharges is characterized by only small variations of the normalized ion temperature gradients, $R / L_{\mathrm{Ti}}$, suggesting a common turbulent transport mechanism such as ITG modes. The evidence presented strongly indicates that these L- and H-mode plasmas are critical gradient limited, showing some degree of profile stiffness. In JET, covering a ion temperature range from 2 to $25 \mathrm{keV}$, this gradient is best described by $R / L_{\mathrm{Ti}}$ depending on the ratio of electron to ion temperature and toroidal rotational shear. However, from the discharges chosen it is not possible to make a distinction which of the two effects prevails. Transport theory suggests that both play a role in the reduction of the plasma turbulence.

ITBs are characterized by an increase of $R / L_{\mathrm{Ti}}$ clearly above the value of $\mathrm{L}$ - and $\mathrm{H}$-modes in both ASDEX Upgrade and JET. In JET, the various types of ITBs, OS, RS plasmas and PEP modes, essentially show the same behaviour. In this context also the PEP mode, which previously was characterized mainly as a particle transport barrier, qualifies as a barrier of the ion heat transport.

No adverse effect of electron heating on the ion transport has been found in JET, applying up to $6 \mathrm{MW}$ additional ICRH in RS ITBs. This is in qualitative agreement with electron heating 
experiments by ECH in ASDEX Upgrade [Wolf_00], although there the local increase of the electron heat flux was much larger. The PEP mode indicates that it is possible to produce ITBs with negative magnetic shear, strong collisional coupling of electrons and ions and thus $T_{\mathrm{e}} \approx T_{\mathrm{i}}$.

The analysis of the $q$-profile during the early evolution of an ion ITB in JET provides a possible explanation for the immediate increase of $R / L_{\mathrm{Ti}}$ observed mainly in RS plasmas. Despite the large uncertainties in the determination of the local magnetic shear at the position of the ITB, the comparison with code calculations of the plasma turbulence suggests that the initial transport reduction is caused by the negative magnetic shear. The role of rational $q$-values in the formation of ITBs has not been considered here [Joffrin_02b, Joffrin_02c, Joffrin_02d].

Although the distinction between L- and H-modes plasmas on the one hand and ITBs on the other, using $R / L_{\mathrm{Ti}}$ as the characteristic quantity, seems to work well, this does not rule out that at least some ITBs can also be limited by critical $R / L_{\mathrm{Ti}}$ values.

The discussion of the effect of density gradients on the ion heat transport is not included in this paper and is left to a later analysis, although there is some evidence that in particular in the JET ELM free H-mode increases of $R / L_{\mathrm{ne}}$ and $R / L_{\mathrm{Ti}}$ are closely linked. Regarding the question whether the ELM free H-mode exhibits ITB phases, the constant $\eta_{\mathrm{i}}$ suggest that it is still critical gradient limited. However, to answer this question more precisely, especially in the context of the effect of density gradients in ITB plasmas, a more detailed analysis is required.

\section{Acknowledgments}

The authors would like to thank C Gowers for providing the JET LIDAR Thomson scattering data, W Dorland for making the GS2 code available and G Pereverzev for the use of the ASTRA code.

\section{References}

[Baker_01] Baker D R et al 2001 Phys. Plasmas 84128

[Becoulet_01] Becoulet A 2001 Plasma Phys. Control. Fusion 43 A395

[Budny_95] Budny R V et al 1995 Nucl. Fusion 351497

[Burrell_97] Burrell K H 1997 Phys. Plasmas 41499

[Challis_01] Challis C D et al 2001 Plasma Phys. Control. Fusion 43861

[Chang_86] Chang C S and Hinton F L 1986 Phys. Fluids 293314

[Connor_77] Connor J W and Taylor J B 1977 Nucl. Fusion 171047

[Connor_94] Connor J W and Wilson H R 1994 Plasma Phys. Control. Fusion 36719

[Dimits_00] Dimits A M et al 2000 Phys. Plasmas 7969

[Eriksson_93] Eriksson L-G, Hellsten T and Willén U 1993 Nucl. Fusion 331037

[Ernst_98] Ernst D R et al 1998 Phys. Rev. Lett. 812454

[Forest_96] Forest C B et al 1996 Phys. Rev. Lett. 773141

[Garbet_01] Garbet X 2001 Plasma Phys. Control. Fusion 43 A251

[Greenfield_99] Greenfield C M et al 1999 Nucl. Fusion 391723

[Guenter_99] Günter S 1999 Nucl. Fusion 391535

[Hawkes_01] Hawkes N C et al 2001 Phys. Rev. Lett. 87115001

[Hawkes_99] Hawkes N C 1999 Rev. Sci. Instrum. 70894

[Hellerman_90] Hellerman M V 1990 Rev. Sci. Instrum. 613479

[Hoang_01] Hoang G T et al 2001 Phys. Rev. Lett. 87125001

[Hobirk_01] Hobirk J et al 2001 Phys. Rev. Lett. 87085002

[Hugon_92] Hugon M et al 1992 Nucl. Fusion 3233

[Huysmans_99] Huysmans G T A et al 1999 Nucl. Fusion 391489 
[Joffrin_02a] Joffrin E et al 2002 Plasma Phys. Control. Fusion 441203

[Joffrin_02b] Joffrin E et al 2002 Nucl. Fusion 42235

[Joffrin_02c] Joffrin E et al 2002 Plasma Phys. Control. Fusion 441739

[Joffrin_02d] Joffrin E et al 2002 19th Conf. on Fusion Energy (Lion, 2002) IAEA-CN-EXP1/13

[Kotschenr_95] Kotschenreuther M, Rewoldt G and Tang W M 1995 Comput. Phys. Commun. 88128

[Lao_85] Lao L L et al 1985 Nucl. Fusion 251611

[Lin_97] Lin Z et al 1997 Phys. Plasmas 41707

[LopezC_95] Lopes Cardozo N J 1995 Plasma Phys. Control. Fusion 37799

[Maget_02] Maget P et al 2002 Statistical analysis of internal transport barriers in JET Nucl. Fusion submitted

[McCarthy_99] Mc Carthy P J 1999 The CLISTE interpretive equilibrium code Rep. 5/85, Max-Planck-Institut für Plasmaphysik, Garching

[Meister_01] Meiter H et al 2001 Nucl. Fusion 411633

[Peeters_00] Peeters A G et al 2000 18th Conf. on Fusion Energy (Sorrento, 2000) IAEA-CN-EXP5/06

[Peeters_02] Peeters A G et al 2002 Nucl. Fusion 421376

[Pereverzev_02] Pereverzev G and Yushmanov P N 2002 ASTRA automated system for transport analysis in a tokamak Rep. 5/98, Max-Planck-Institut für Plasmaphysik, Garching

[Petty_02] Petty C C et al 2002 Phys. Plasmas 9128

[Rimini_99] Rimini F G et al 1999 Nucl. Fusion 391591

[Ryter_01] Ryter F et al 2001 Phys. Rev. Lett. 865498

[Sips_98] Sips A C C et al 1998 Plasma Phys. Control. Fusion 401171

[Staebler_98] Staebler G M 1998 et al 25th Eur. Conf. on Plasma Physics and Controlled Fusion (Praha, 1998) vol 22C (European Physical Society) p 2006

[Suttrop_01] Suttrop W et al 2001 28th Eur. Conf. on Plasma Physics and Controlled Fusion (Madeira, 2001) (European Physical Society) P3.016

[Tardini_02] Tardini G et al 2002 Nucl. Fusion $\mathbf{4 2} 258$

[Tresset_02] Tresset et al 2002 Nucl. Fusion 42520

[Wakatani_98] Wakatani M 1998 Plasma Phys. Control. Fusion 40597

[Waltz_94] Waltz R E et al 1994 Phys. Plasmas 12229

[Waltz_95] Waltz R E et al 1995 Phys. Plasmas 22408

[Waltz_98] Waltz R E et al 1998 Phys. Plasmas 51784

[Wolf_00] Wolf R C et al 2000 Phys. Plasmas 71839

[Wolf_03] Wolf R C 2003 Plasma Phys. Control. Fusion R1

[Wolf_97] Wolf R C et al 1997 24th Eur. Conf. on Plasma Physics and Controlled Fusion (Berchtesgaden, 1997) (European Physical Society) p 1509

[Wolf_99] Wolf R C et al 1999 Plasma Phys. Control. Fusion 41 B93 